\title{
Comparative susceptibilities of various AIDS- associated and human urogenital tract mycoplasmas and strains of Mycoplasma pneumoniae to 10 classes of antimicrobial agent in vitro
}

\author{
P. C. T. HANNAN
}

Mycoplasma Experience Ltd 1 Norbury Road, Reigate, Surrey RH2 $9 B Y$

\begin{abstract}
The susceptibilities of 40 strains of various Mycoplasma species to 10 classes of antimicrobial agents were compared in vitro by a broth microdilution method. The strains tested comprised 20 strains of four AIDS-associated species - M. penetrans (1 strain), M. fermentas (5 strains), M. pirum (6 strains) and $M$. genitalium (8 strains) nine strains of the urogenital tract species $M$. hominis and 11 strains of $M$. pneumoniae. The results demonstrated wide variation in the susceptibilities of the different Mycoplasma spp. to different classes of antimicrobial agent. All the mycoplasmas were susceptible or highly susceptible to the fluoroquinolones, with sparfloxacin the most active, and to the diterpine antibiotic tiamulin. $M$. pneumoniae and $M$. genitalium strains were also highly susceptible to the macrolides, particularly azithromycin and had similar antibiotic susceptibility patterns to most other antimicrobial agents. However, all strains of $M$. genitalium were resistant to streptomycin (MIC $250->500 \mathrm{mg} / \mathrm{L}$ ) whereas all $M$. pneumoniae isolates, except the MAC strain, were susceptible (MICs 1.25$12.5 \mathrm{mg} / \mathrm{L}$ ). M. pirum isolates varied considerably in their susceptibility to macrolides (MIC range versus azithromycin $0.0025->100 \mathrm{mg} / \mathrm{L}$ ). M. fermentans strains were susceptible to the tetracyclines, lincosamides and mupirocin, but varied in susceptibility to aminoglycosides. Most $M$. hominis strains were susceptible to the tetracyclines and all were susceptible to clindamycin and mupirocin. M penetrans GTU 54 was susceptible to azithromycin, the tetracyclines and lincosamides as well as to the fluoroquinolones and tiamulin.
\end{abstract}

\section{Introduction}

Over the past decade various species of Mycoplasma have been cultured from or detected in body fluids or tissues from AIDS patients or HIV-seropositive individuals. Strains of $M$. fermentans, M. penetrans and $M$. pirum have all been cultured during such studies [1-6] and $M$. genitalium has been detected by the polymerase chain reaction [5], leading to speculation that they might act as co-factors in the pathogenesis of AIDS.

The highly fastidious urogenital tract species $M$. genitalium has also been isolated in culture from the throats of non-AIDS patients in association with $M$.

Received 20 Jan. 1998; revised version accepted 30 March 1998.

Corresponding author: Dr P. C. T. Hannan. pneumoniae [7], although it is unclear whether $M$. genitalium plays any role in respiratory disease. However, as $M$. genitalium and $M$. pneumoniae share many properties - including the presence of an organised tip attachment structure, similar biochemical characteristics, adherence capabilities and serological cross-reactivities - differentiation in the clinical laboratory is difficult $[8,9]$. Attempts to detect antibiotics with selective activity against one or other of these species, which could aid in their differentiation, have so far been unsuccessful [8].

The potential importance of mycoplasmas in AIDS, their role in other human infections and their involvement as cell-culture contaminants has led to studies of the antimicrobial susceptibilities of some of these species $[8,10-25]$. This report extends those investigations, compares the antimicrobial susceptibilities of $M$. genitalium and $M$. pneumoniae strains and 
includes MIC data on recent isolates of $M$ hominis from human infections.

\section{Materials and methods}

\section{Mycoplasma cultures}

The type strains $M$. fermentans PG 18 (NCTC 10117), M. hominis PG21 (NCTC 10111), M. pirum HRC 70159 (NCTC 11702), $M$. pneumoniae FH (NCTC 10119), $M$. genitalium G37 (NCTC 10195) and $M$. penetrans GTU 54 (ATCC 55252) were obtained either from the National Collection of Type Cultures (NCTC), 61 Colindale Avenue, London or the American Type Culture Collection (ATCC), 12301 Parklawn Drive, Rockville, MD 20852, USA.

M. fermentans incognitus was supplied by Dr S-C. Lo (Department of Defense, Armed Forces Institute of Pathology, Washington, DC, USA) and was isolated during transfection studies with DNA extracted from Kaposi's sarcoma tissue from an AIDS patient [1]. $M$. fermentans strain nos. 5 and 29, isolated in cell-free medium from urine sediments of AIDS patients [3], were obtained from Dr R. B. Kundsin (Brigham Women's Hospital, Harvard Medical School, Boston, MA, USA). Professor L. Montagnier (Institute Pasteur, Paris, France) kindly supplied $M$. fermentans strain AOU which was cultured from peripheral blood mononuclear cells (PBMCs) purified from the blood of an AIDS patient [2].

Six recent isolates of $M$. hominis (M443/90, M411/ 90, M286/93, M329/92, M265/92 and M680/90) from infected hospitalised patients in the UK, and strain $\mathrm{H} 27$ isolated from the urethra of a male with acute Reiter's syndrome in 1962 were supplied by $\mathrm{Dr}$ D. Pitcher (Central Public Health Laboratory, 61 Colindale Avenue, London). Dr J. Fabbri (Kantonsspital, Basel, Switzerland) supplied $M$. hominis strain Basel isolated from a Swiss patient with pneumonia. Five previously documented strains of $M$. pirum isolated from PBMCs from AIDS patients (COL, BER, RAO, VUC and NOU) [5] were supplied by Dr O. Grau (Institute Pasteur) and strain ICRF (UK cell culture isolate) by Helena M. Windsor (Mycoplasma Experience Ltd, Reigate, Surrey). These strains were tested against a wide selection of antimicrobial agents. Dr J. G. Tully (Mycoplasma Section, NIAID, Frederick Cancer Research Facility, Frederick, MD, USA) provided four additional American (1901672, 1890758, 1902677 and FD870) and one British (MAD-T) lyophilised cell-culture isolates of $M$. pirum, identified and described previously [26]. These strains were used only to confirm the macrolide susceptibilities of $M$. pirum cell-culture isolates.

Low passage isolates of $M$. pneumoniae (strains M $192 / 77$ and $M 195 / 77$ ) from patients with primary atypical pneumonia (PAP) were supplied by $\mathrm{Dr}$.
Leach (Mycoplasma reference Facility, NCTC, Central Public Health Laboratory, 61 Colindale Avenue, London). Dr D. Pitcher supplied a further five strains of M. pneumoniae (M 336/81, M163/95, M 113/95, M $167 / 95$ and $M$ 1150/96) and Dr G. Taylor (Clinical Research Centre, Northwick Park Hospital, Harrow), strain 427a. M. pneumoniae strains MAC (ATCC 15492) and 1428 (ATCC 29085) were obtained from the ATCC. All these strains were tested for susceptibility to a wide range of antimicrobial agents. Additional $M$. pneumoniae strains M 289/67, M 129-B7, M 129-B170 and M 36/92 (supplied by Dr D. Pitcher) and $M 950 / 81, M 1598 / 81, M$ 2659/82, M 191/83 (obtained from Dr M. Sillis, Virology Department, Public Health Laboratory, Bowthorpe Road, Norwich) and UTMB-10P (ATCC 49894) supplied by the ATCC, were tested only to confirm their susceptibilities to streptomycin. $M$. genitalium strain UTMB-10G (ATCC 49899) which was isolated in mixed culture with $M$. pneumoniae UTMB-10P from the synovial fluid from a patient with PAP and subsequent polyarthritis [27] was obtained, freeze-dried, from the ATCC. M. genitalium strains M2288, M2300, M2321 and M2341, cited and identifed previously [28], were supplied by Dr J. S. Jensen (Mycoplasma Laboratory, Neisseria Department, Statens Seruminstitut, DK 2300, Copenhagen, Denmark) and were isolated by cell culture from urethral swabs from Danish men with urethritis. $M$. genitalium strains M30 and 12959 (a low passage strain of the G37 strain) were isolated from the urethras of male patients with non-gonococcal urethritis by Dr P. M. Furr (St Mary's Hospital, Paddington, London).

The identities of the $M$. fermentans, M. hominis, $M$. pneumoniae and $M$. pirum strains were confirmed serologically by the growth inhibition test [29] with species-specific antisera. Antisera to $M$. genitalium G37 inhibited the reference strain and all the donated $M$. genitalium strains, but showed some cross-reaction with $M$. pneumoniae strains $\mathrm{FH}$ and 1428. $M$. penetrans GTU 54, for which no growth inhibiting antiserum was available, was used within three in-vitro passages after recovery from the freeze-dried state. All strains were cultured on inhibitor-free mycoplasmal agar and were found to be free from bacterial contamination.

\section{Mycoplasma culture media}

A complete, commercially available, quality controlled, liquid medium (Mycoplasma Experience Ltd) containing serum and fresh yeast extract and either glucose $0.1 \% \mathrm{w} / \mathrm{v}$ at $\mathrm{pH} 7.6$ for the glucose fermenting mycoplasmas ( $M$. fermentans, $M$. penetrans, $M$. pirum, $M$. pneumoniae and $M$. genitalium) or arginine $0.1 \% \mathrm{w} / \mathrm{v}$ at $\mathrm{pH} 6.8$ for $M$. hominis (arginine cataboliser) and phenol red $0.004 \%$, was used in the cultivation of the mycoplasmas and in MIC tests. 
Growth of the glucose-fermenting mycoplasmas was denoted by a change in colour of the medium from pink to orange/yellow and that of $M$. hominis from orange/yellow to pink.

\section{Preparation of stock cultures}

All mycoplasma strains were grown aerobically at $36^{\circ} \mathrm{C}$ in mycoplasma broth in sterile plasitc 5-ml screw-capped vials until an acid or alkaline colour shift occurred. After addition of sterile glycerol $5 \% \mathrm{w} / \mathrm{v}$ the cultures were dispensed in $1-\mathrm{ml}$ aliquots and frozen at $-70^{\circ} \mathrm{C}$. These were used to initiate further broth cultures, which, after the addition of $4 \mathrm{ml}$ of fresh broth medium and incubation until a definite colour change occurred, were titrated in sealed microdilution trays to determine the number of colour changing units (ccu) after a recorded period of incubation. Replication of this procedure allowed the preparation of inocula which were then further diluted in mycoplasma broth to give between $10^{3}$ and $10^{5} \mathrm{ccu} / \mathrm{ml}$ for use in MIC tests.

\section{Sources and preparation of antimicrobial agents}

With the exception of the following, all compounds were supplied by Sigma. Sparfloxacin (technical grade) and azithromycin (technical grade) were supplied by Rhône Poulenc Rorer, Paris, France and Pfizer Central Research, Sandwich, Kent, respectively. Tiamulin hydrogen fumarate was supplied by Sandoz, and mupirocin lithium salt and clarithromycin by Smith Kline Beecham. Sterile solutions of ciprofloxacin (Ciproxin, Bayer) and netilmicin (Netillin, ScheringPlough) were purchased and diluted for use in MIC tests. Most of the compounds were dissolved in deionised water to achieve a concentration of $1 \mathrm{mg} / \mathrm{ml}$ and were sterilised by membrane filtration $(0.2 \mu \mathrm{m}$ pore size). The aminoglycosides were prepared at a concentration of $4 \mathrm{mg} / \mathrm{ml}$. Erythromycin, clarithromycin and aztithromycin were dissolved in aqueous ethanol $10 \% \mathrm{v} / \mathrm{v}$. Ofloxácin and sparfloxacin were first dissolved in sterile $0.1 \mathrm{~N} \mathrm{NaOH}$ and were then made up to volume with de-ionised water before sterilisation. Further compound dilutions were prepared in sterile plastic or glass vials at double the required concentration in mycoplasma broth to allow for a 1:1 dilution by the mycoplasma inoculum in MIC tests.

The dilution series of antimicrobial agents used included both 10 -fold and doubling dilutions to minimise dilution errors and to reduce excessive compound 'carry over'. The dilution ranges used to test particular mycoplasma-antimicrobial combinations varied and were selected either with prior knowledge of the likely MIC or after siting experiments. Thus, $M$. fermentans strains, some of which have been shown previously to be resistant to aminoglycosides [11], were tested against a range of concentrations for this class of compound from 500 to $0.25 \mathrm{mg} / \mathrm{L}$, whereas $M$. pneumoniae strains, which are known to be highly sensistive to macrolides [8], were tested against a much lower range, e.g., $1-0.0001 \mathrm{mg} / \mathrm{L}$, as were the $M$. genitalium strains. Other dilution ranges used for various mycoplasmal-antimicrobial combinations were $100-0.1 \mathrm{mg} / \mathrm{L}$ and $10-0.01 \mathrm{mg} / \mathrm{L}$.

\section{$M I C$ tests}

MIC tests were performed in 96-well, microdilution sensititre plates by a modification of the method described by Tanner and $\mathrm{Wu}$ [30]. The compound dilutions were mixed with an equal volume $(100 \mu 1)$ of the challenge inocula containing $10^{3}-10^{5} \mathrm{ccu} / \mathrm{ml}$ in microtitration wells. Each sensititre plate contained uninoculated medium as a sterility control, medium at $\mathrm{pH} 6.8$ or $\mathrm{pH} 7.6$ as end-point controls (depending on whether the $\mathrm{pH}$ shift was to an acid or alkaline reaction) and drug-free inoculated growth controls. To confirm the challenge inoculum titres, $100 \mu \mathrm{l}$ of dilutions of the challenge inoculum $\left(10^{1}-10^{5}\right)$ were added to $100 \mu \mathrm{l}$ of medium in sensititre wells. The plates were then sealed with adhesive film (Falcon) and were incubated at $36^{\circ} \mathrm{C}$ until the colour in the growth control well matched that of the end-point control. The incubation periods varied according to the Mycoplasma species, the faster growing species taking 2-3 days to cause a colour change and the slower growers such as $M$. genitalium up to 7 days. Initial MICs were recorded when the colour of the growth control matched that of the end-point control, the MIC being the lowest drug concentration to show no change in colour. Final MICs were recorded 7 days later and were the lowest drug concentration in which no colour change was seen. Incubation of the plates containing dilutions of the challenge inocula was continued until colour changes were complete. If MIC end-points were not achieved, or if the mycoplasma challenge fell outside $10^{3}-$ $10^{5} \mathrm{ccu} / \mathrm{ml}$, tests were repeated.

\section{Results}

The in-vitro susceptibilities of the six mycoplasma species examined to 10 classes of antimicrobial agents are shown in Table 1. Sparfloxacin and the veterinary antibiotic tiamulin showed the broadest spectra of antimycoplasmal activities with MICs of sparfloxacin ranging from $0.0025-0.1 \mathrm{mg} / \mathrm{L}$ against $M$. pneumoniae to $0.1-0.5 \mathrm{mg} / \mathrm{L}$ against strains of $M$. genitalium. MICs for tiamulin ranged from $0.0025-0.01 \mathrm{mg} / \mathrm{L}$ against $M$. genitalium to $0.025-0.1 \mathrm{mg} / \mathrm{L}$ against $M$. fermentans.

The macrolides were particularly active in vitro against $M$. genitalium and $M$. pneumoniae strains, azithromycin being the most active of this class of compound (initial MICs against $M$. genitalium $0.0005-0.001 \mathrm{mg} / \mathrm{L}$ and against $M$. pneumoniae $0.001-0.005 \mathrm{mg} / \mathrm{L}$ ). The macrolides were relatively 


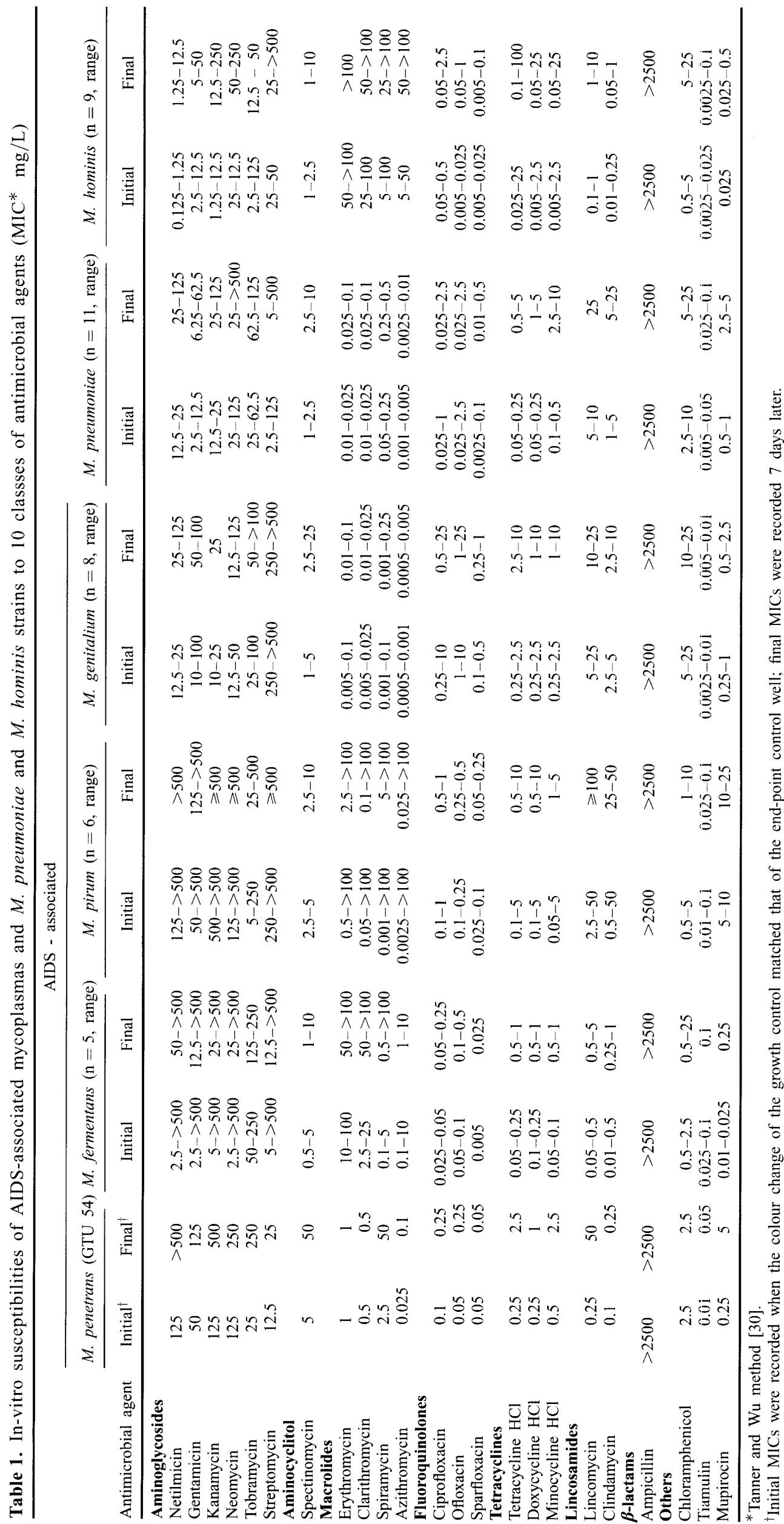


inactive against the other Mycoplasma species tested; some strains of $M$. pirum, $M$. hominis and $M$. fermentans were resistant to $\geqslant 100 \mathrm{mg} / \mathrm{L}$ of these agents. $M$. pirim strains isolated from PBMCs purified from the blood of French AIDS patients were highly resistant to erythromycin, clarithromycin, spiramycin and azithromycin, whereas the type strain and another tissue culture cell isolate (ICRF) were sensitive. MIC tests on additional American and British $M$. pirum strains isolated from contaminated tissue culture cells revealed wide variation in susceptibility to macrolides (Table 2).

The tetracyclines showed good to moderately good in-vitro activity against $M$. penetrans GTU-54 (initial MIC range $0.25-0.5 \mathrm{mg} / \mathrm{L}$ ), $M$. fermentans (initial MIC range $0.05-0.25 \mathrm{gm} / \mathrm{L}$ ), $M$. pneumoniae (initial MIC range $0.05-0.5 \mathrm{mg} / \mathrm{L}$ ) and $M$. hominis (initial MIC range $0.005-25 \mathrm{mg} / \mathrm{L}$ ), but were slightly less effective against $M$. genitalium (initial MIC range $0.25-2.5 \mathrm{mg} / \mathrm{L}$ ) (Table 1). M. hominis strain Basel was moderately resistant to tetracycline $\mathrm{HCl}$ (MIC $25 \mathrm{mg} / \mathrm{L}$ ).

The aminoglycosides were mainly inactive in vitro against the majority of the AIDS-associated and urogenital tract mycoplasmas tested. Some strains of $M$. fermentans, $M$. pirum and $M$. penetrans showed overall aminoglycoside resistance of $125->500 \mathrm{mg} / \mathrm{L}$. The type strain of $M$. fermentans (PG-18) and two strains of $M$. fermentans isolated from urine deposits from AIDS patients without the use of cell cultures were - with the exception of tobramycin - aminoglycoside-sensitive; two other AIDS isolates ( $M$. fermentans strains incognitus and AOU), obtained from cultured cells, were aminoglycoside resistant (Table 3). All strains of $M$. genitalium were highly resistant to streptomycin (initial MIC range 250$>500 \mathrm{mg} / \mathrm{L}$ ) irrespective of whether cell cultures were used in the isolation process, whereas with the exception of $M$. pneumoniae strain MAC (initial MIC $125 \mathrm{mg} / \mathrm{L}$ ), all the $M$. pneumoniae strains were streptomycin-sensitive (initial MIC range 2.5$12.5 \mathrm{mg} / \mathrm{L}$ ). MIC tests on a further nine $M$. pneumoniae strains, including strain UTMB-10P which was isolated in mixed culture with $M$. genitalium UTMB-10G from an arthritic joint of a patient with PAP, substantiated these findings (Table 4).

Clindamycin showed good in-vitro activity against $M$. hominis (initial MICs $0.01-0.25 \mathrm{mg} / \mathrm{L}$ ) and $M$. fermentans strains (initial MICs $0.01-0.5 \mathrm{mg} / \mathrm{L}$ ) (Table 1). Final MICs of most of the compounds examined were generally 2-10-fold higher than the initial MICs, suggesting that most classes of antimicrobial agent were predominantly mycoplasmastatic. However, the degree of difference between the initial MICs and the final MICs varied from compound to compound, both within particular classes of antibiotic and between the different antibiotic classes themselves

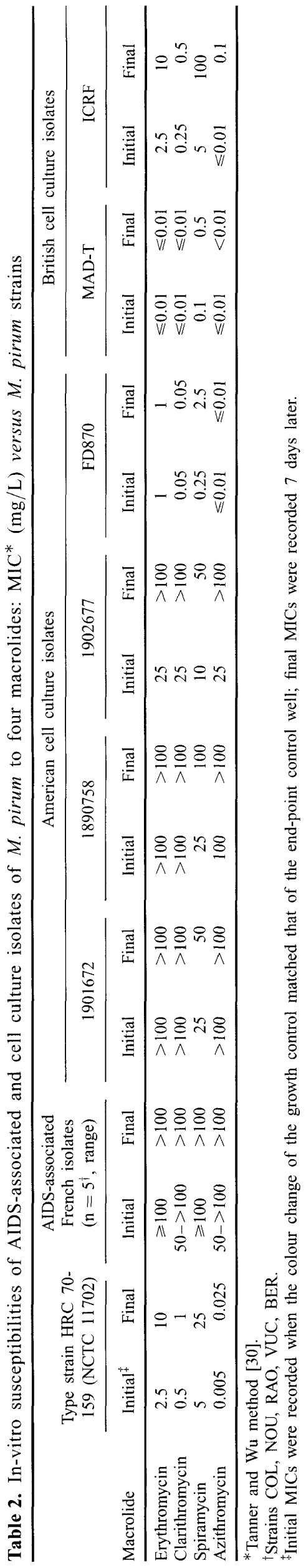


Table 3. The susceptibilities of four AIDS-associated strains of $M$. fermentans to six aminoglycoside antibiotics compared with that of the type strain PG18 (MIC* $\mathrm{mg} / \mathrm{L}$ )

\begin{tabular}{|c|c|c|c|c|c|c|c|c|c|c|}
\hline \multirow[b]{3}{*}{ Aminoglycoside } & \multicolumn{6}{|c|}{ Isolated in cell-free media } & \multicolumn{4}{|c|}{ Isolated with cultured cells } \\
\hline & \multicolumn{2}{|c|}{$\begin{array}{l}\text { Type strain } \\
\text { PG-18 }\end{array}$} & \multicolumn{2}{|c|}{$\begin{array}{l}\text { Strain } 5 \text { from } \\
\text { AIDS urine }\end{array}$} & \multicolumn{2}{|c|}{$\begin{array}{l}\text { Strain } 29 \text { from } \\
\text { AIDS urine }\end{array}$} & \multicolumn{2}{|c|}{$\begin{array}{l}\text { AIDS Kaposi's } \\
\text { sarcoma DNA } \\
\text { incognitus }\end{array}$} & \multicolumn{2}{|c|}{$\begin{array}{l}\text { AIDS PBM } \\
\text { cells }^{\dagger} \text { AOU }\end{array}$} \\
\hline & Initial $^{+}$ & Final & Initial & Final & Initial & Final & Initial & Final & Initial & Final \\
\hline Netilmicin & 2.5 & 50 & 12.5 & 125 & 12.5 & 125 & $>500$ & $>500$ & $>500$ & $>500$ \\
\hline Gentamicin & 2.5 & 12.5 & 12.5 & 25 & 12.5 & 25 & $>500$ & $>500$ & $>500$ & $>500$ \\
\hline Kanamycin & 5 & 25 & 12.5 & 25 & 12.5 & 25 & $>500$ & $>500$ & $>500$ & $>500$ \\
\hline Neomycin & 25 & 50 & 50 & 125 & 50 & 125 & $>500$ & $>500$ & $>500$ & $>500$ \\
\hline Tobramycin & 50 & 125 & 125 & 250 & 125 & 250 & 250 & 250 & 125 & 250 \\
\hline Streptomycin & 5 & 12.5 & 12.5 & $>500$ & 12.5 & $>500$ & $>500$ & $>500$ & 500 & $>500$ \\
\hline
\end{tabular}

* Tanner and $\mathrm{Wu}$ method [30].

PBMC cultures from the blood of an AIDS patient.

"Initial MICs were recorded when the colour change of the growth control matched that of the end-point control well; final MICs were recorded 7 days later.

Table 4. In-vitro susceptibilities of $11 \mathrm{M}$. pneumoniae and two $M$. genitalium strains to streptomycin

\begin{tabular}{lcc}
\hline & \multicolumn{2}{c}{ MIC* $^{*}(\mathrm{mg} / \mathrm{L})$} \\
\cline { 2 - 3 } Strain & Initial $^{\dagger}$ & Final \\
\hline M. pneumoniae & & \\
Type strain FH (NCTC 10119) & $12.5^{\ddagger}$ & $25^{\ddagger}$ \\
MAC (ATCC 15492) & $125.0^{\ddagger}$ & $500^{\ddagger}$ \\
Additional strains & & \\
M129-B7 (ATCC 29342) & 1.25 & 2.5 \\
M129-B170 (ATCC 29343) & 2.5 & 12.5 \\
M289/67 & 2.5 & 12.5 \\
M950/81 & 2.5 & 5 \\
M1598/82 & 2.5 & 5 \\
M191/83 & 2.5 & 5 \\
M36/92 & 1.25 & 5 \\
M2659/82 & 2.5 & 5 \\
UTMB-10P (ATCC 49894) & $2.5^{\ddagger}$ & $12.5^{\ddagger}$ \\
Range (additional strains) & $1.25-2.5$ & $2.5-12.5$ \\
M. genitalium & & \\
Type strain G37 (NCTC 10195) & $>500^{\ddagger}$ & $>500^{\ddagger}$ \\
UTMB-10G (ATCC 49899) & $\geqslant 500^{\ddagger}$ & $\geqslant 500^{\ddagger}$ \\
\hline
\end{tabular}

*Tanner and Wu method [30].

${ }^{\dagger}$ Initial MICs were recorded when the colour change of the growth control matched that of the end-point control well; final MICs were recorded 7 days later.

${ }^{\ddagger}$ Results of duplicate tests.

and was influenced by the Mycoplasma sp. under investigation. The fluoroquinolones, which are known to be mycoplasmacidal in vitro [31], had final MIC values only slightly higher than the initial MICs.

\section{Discussion}

Although $M$. pneumoniae, $M$. fermentans and $M$. hominis have been studied extensively, there are few reports on the antimicrobial susceptibilites of $M$. penetrans, $M$. pirum and $M$. genitalium. Hayes et al. examined nine strains of $M$. penetrans isolated from either the urine or semen of AIDS patient [12], and Poulin et al. investigated the susceptibilities of the type strains of $M$. pirum (HRC 70-159) and $M$. penetrans (GTU-54) together with certain strains of $M$. fermentans [23]. The antimicrobial susceptibilities of seven
M. genitalium strains have also been reported recently [8].

The results reported in the current study are in general agreement with those of previous studies in which the same species were investigated, although different MIC methods were used by different researchers Those who used freshly thawed inocula [11-13] or cultures in the lag phase [17-22] tended to obtain somewhat lower MICs for some compounds than those reported in the current study, in which mycoplasmas in the logarithmic growth phase were used. Those studies that used agar dilution methods obtained MICs similar to or slightly higher than those recorded in the present study $[8,18-21,24]$.

The current results clearly demonstrate the wide variation in susceptibilities of AIDS-associated and human urogenital tract and respiratory mycoplasmas to different classes of antibiotic. Sparfloxacin had excellent in-vitro activity against all the Mycoplasma spp. tested and, therefore, has significant potential for treating mycoplasma infections in AIDS patients and infections caused by urogenital tract mycoplasmas and M. pneumoniae in non-AIDS patients. Tiamulin also had a broad spectrum of good anti-mycoplasmal activity in vitro, but is not licensed for human use. However, both these compounds might offer alternative means of eradicating human mycoplasmas from tissue culture cells if used under controlled conditions. Ciprofloxacin has already been recommended for this purpose [32].

The excellent in-vitro anti-mycoplasmal activities recorded here for the macrolides, particularly azithromycin, against $M$. genitalium and $M$. pneumoniae strains have also been observed by others [8], as has the similarity between the antimicrobial susceptibility profiles of these two Mycoplasma spp. [8]. However, the marked difference in their susceptibility to streptomycin has not been reported previously. In the current study, all the $M$. genitalium strains were highly 
resistant to streptomycin, while all strains of $M$. pneumoniae, with the exception of the MAC strain, were susceptible. In particular, $M$. pneumoniae UTMB-10P and $M$. genitalium UTMB-10G, which were isolated together from synovial fluid of the same patient, were streptomycin-sensitive and streptomycin-resistant, respectively. It seems likely that streptomycin resistance in the MAC strain may have resulted from its reported passage in chick embryos containing streptomycin $50 \mathrm{mg} / \mathrm{L}$ during its primary isolation $[33,34]$ as repeated passage in the presence of streptomycin in vitro has been shown to induce streptomycin resistance in $M$. pneumoniae [35]. Streptomycin resistance ( $\geqslant 100 \mathrm{mg} / \mathrm{L}$ ) has also been reported in some Japanese $M$. pneumoniae strains isolated between 1965 and 1975 [36], when streptomycin was still in use for the treatment of tuberculosis and other respiratory infections. However, now that streptomycin has been superseded by more active and safer antibiotics and is used only infrequently, streptomycin resistance would not be expected to be present in current $M$. pneumoniae isolates. Gentamicin and the other two deoxystreptamine-containing aminoglycosides do not induce cross-resistance to streptomycin (streptidine-containing aminoglycoside [11, 37]). Therefore, it would seem that streptomycin resistance could by used as a marker for differentiating $M$. genitalium from recent isolates of $M$. pneumoniae and that inclusion of streptomycin at concentrations inhibitory to $M$. pneumoniae might form the basis for developing a selective medium for $M$. genitalium in respiratory infections.

Until recently $M$. pirum had only been isolated from mycoplasma-contaminated cell cultures and its natural host was unknown. However, the recent report by De Barbeyrac et al. [6] that $M$. pirum had been cultured from the throats and urine of AIDS patients without the use of cell cultures seems to confirm that $M$. pirum is a human Mycoplasma sp. The observation in the present study that the type strain HRC 70-159, the two British cell culture isolates MAD-T and ICRF and the American cell culture isolate FD 870 are macrolide-sensitive, while the other American cell culture strains are resistant, suggests that this class of antibiotic has been used in some laboratories to combat bacterial or mycoplasmal contamination in tissue culture cell lines and, therefore, may be responsible for inducing macrolide resistance in certain strains of $M$. pirum. The reason for the resistance of the French AIDS-associated $M$. pirum strains is unclear, as macrolides were not included in the media used to cultivate the PBMCs from the blood of the AIDS patients (O. Grau, personal communication).

The complete resistance of the AIDS-associated $M$. fermentans strain incognitus and $M$. fermentans cell culture isolates to aminoglycosides and the aminoglycoside susceptibility of $M$. fermentans strains isolated from patients without the use of eukaryotic cells has been reported previously $[11,38]$. This has been used as an argument for concluding that the incognitus strain was probably a cell culture contaminant. The current observation that $M$. fermentans AOU, isolated from PBMCs purified from the blood of an AIDS patient, was also aminoglycoside-resistant, while strains 5 and 29, recovered from the urine of AIDS patients in cell-free medium, were aminoglycosidesensitive, suggests that strain AOU also probably originated from cultured cells rather than from the AIDS patient. This suggestion is supported by a recent observation that the AOU strain is closely related to a Japanese mouse cell culture isolate (F-7) when compared by the newly developed pyrolysis mass spectrometry method for $M$. fermentans [39].

The author thanks all those who supplied mycoplasma cultures enabling this study to be carried out, Rhône Poulenc Rorer for the sample of sparfloxacin, Pfizer Central Research for azithromycin, Sandoz for the sample ot tiamulin and Smith Kline Beecham for supplying mupirocin and clarithromycin. Thanks are also extended to Dr G. D. Windsor and Helena M. Windsor at Mycoplasma Experience Ltd, UK for supplying the facilities, media and apparatus used in this study, to Dr D. Pitcher for supplying the $M$. genitalium and $M$. pirum growth inhibiting antisera and to Mrs $\mathrm{S}$. Hayes for typing the manuscript.

\section{References}

1. Lo SC, Shih JW-K, Yang N-Y, Ou C-Y, Wang RY-H. A novel virus-like infectious agent in patients with AIDS. Am J Trop Med Hyg 1989; 40: 213-226.

2. Montagnier L, Berneman D, Guétard D et al. Inhibition de l'infectionsité de souches prototypes du VIH par des anticorps dirigés contre une séquence peptidique de mycoplasme. $C R$ Acad Sci 1990; Paris t 311, Série 111: 425-430.

3. Dawson MS, Hayes MM, Wang RY-H, Armstrong D, Kundsin RB, Lo SC. Detection and isolation of Mycoplasma fermentans from urine of human immunodeficiency virus type-1 infected patients. Arch Pathol Lab Med 1993; 117: $511-514$.

4. Lo S-C, Hayes MM, Wang RY-H, Pierce PF, Kotani H, Shih JW-K. Newly discovered mycoplasma isolated from patients infected with HIV. Lancet 1991; 338: 1415-1418.

5. Grau O, Kovacic R, Griffais R, Montagnier L. Development of a selective and sensitive polymerase chain reaction assay for the detection of Mycoplasma pirum. FEMS Microbiol Lett 1993; 106: 327-334.

6. De Barbeyrac B, Dupon M, Grau O et al. Mycoplasmas in patients infected with HIV-1. IOM Letters vol. 4. 11th International Congress of the International Organisation of Mycoplasmology (IOM), Orlando, FL, USA. July 14-19, 1996: 69.

7. Baseman JB, Dallo SF, Tully JG, Rose DL. Isolation and characterization of Mycoplasma genitalium strains from the human respiratory tract. $J$ Clin Microbiol 1988; 26: 2266-2269.

8. Renaudin H, Tully JG, Bébéar C. In vitro susceptibilities of Mycoplasma genitalium to antibiotics. Antimicrob Agents Chemother 1992; 36: 870-872.

9. Lind $\mathrm{K}$, Lindhardt $\mathrm{B} \emptyset$, Schütten HJ, Blom J, Christiansen C. Serological cross-reactions between Mycoplasma genitalium and Mycoplasma pneumoniae. J Clin Microbiol 1984; 20: 1036-1043.

10. Hayes MM, Foo $\mathrm{H}-\mathrm{H}$, Kotani $\mathrm{H}$, Wear DJ, Lo S-C. In vitro antibiotic susceptibility testing of different strains of $\mathrm{Myco}$ plasma fermentans isolated from a variety of sources. Antimicrob Agents Chemother 1993; 37: 2500-2503.

11. Hannan PCT. Antibiotic susceptibility of Mycoplasma fermentans strains from various sources and the development of resistance to aminoglycosides in vitro. J Med Microbiol 1995; 42: $421-428$

12. Hayes MM, Foo $\mathrm{H}-\mathrm{H}$, Timenetsky J, Lo S-C. In vitro antibiotic sensitivity testing of clinical isolates of Mycoplasma penetrans 
from patients with AIDS. Antimicrob Agents Chemother 1995; 39: $1386-1387$

13. Jao RL, Finland M. Susceptibility of Mycoplasma pneumoniae to 21 antibiotics in vitro. Am J Med Sci 1967; 253:639-650.

14. Stewart SM, Burnet ME, Young JE. In-vitro sensitivity of strains of Mycoplasmas from human sources to antibiotics and to sodium aurothiomalate and tylosin tartrate. I Med Microbiol 1969; 2: 287-292

15. Braun P, Klein JO, Kass EH. Susceptibility of genital mycoplasmas to antimicrobial agents. Appl Microbiol 1970; 19: $62-70$.

16. Quentin C, Cantet P, Renaudin H, Bébéar C. [Antibiotic sensitivity of mycoplasmas pathogenic for man.] Sensibilité aux antibiotiques des mycoplasmes pathogenes pour l'homme. Pathol Biol 1985; 33: 205-212.

17. Waites KB, Cassell GH, Canupp $\mathrm{KC}$, Fernandes $\mathrm{PB}$. In vitro susceptibilities of mycoplasmas and ureaplasmas to new macrolides and aryl-fluoroquinolones. Antimicrob Agents Chemother 1988; 32: 1500-1502.

18. Kenny GE, Hooton TM, Roberts MC, Cartwright FD, Hoyt J. Susceptibilities of genital mycoplasmas to the newer quinolones as determined by the agar dilution method. Antimicrob Agents Chemother 1989; 33: 103-107.

19. Kenny GE, Cartwright FD. Susceptibilities of Mycoplasma hominis and Ureaplasma urealyticum to two new quinolines, Sparfloxacin and WIN 57273. Antimicrob Agents Chemother 1991; 35: $1515-1516$.

20. Kenny GE, Cartwright FD. Susceptibilities of Mycoplasma hominis, Mycoplasma pneumoniae, and Ureaplasma urealyticum to new glycyclines in comparison with those to older tetracyclines. Antimicrob Agents Chemother 1994; 38: $2628-2632$.

21. Kenny GE, Cartwright FD. Susceptibility of Mycoplasma pneumoniae to several new quinolones, tetracycline, and erythromycin. Antimicrob Agents Chemother 1991; 35: $587-589$.

22. Waites KB, Duffy LB, Schmid T, Crabb D, Pate MS, Cassell $\mathrm{GH}$. In vitro susceptibilities of Mycoplasma pneumoniae. Mycoplasma hominis, and Ureaplasma urealyticum to Sparfloxacin and PD 127391. Antimicrob Agents Chemother 1991; 35: $1181-1185$

23. Poulin SA, Perkins RE, Kundsin RB. Antibiotic susceptibilites of AIDS-associated Mycoplasmas. J Clin Microbiol 1994; 32: 1101-1103.

24. Furneri PM, Bisignano G, Cerniglia G, Nicoletti G, Cesana M, Tempera G. In vitro antimycoplasmal activities of Rufloxacin and its metabolite MF 922. Antimicrob Agents Chemother 1994; 38: 2651-2654.
25. Bébéar CM, Renaudin H, Aydin MD, Chantot JF, Bébéar C. Invitro activity of ketolides against mycoplasmas. $J$ Antimicrob Chemother 1997; 39: 669-670.

26. Del Guidice RA, Tully JG, Rose Dl, Cole RM. Mycoplasma pirum sp. nov., a terminal structure mollcute from cell cultures. Int J Syst Bacteriol 1985; 35: 285-291.

27. Tully JG, Rose DL, Baseman JB, Dallo SF, Lazzell AL, Davis CP. Mycoplasma pneumoniae and Mycoplasma genitalium mixture in synovial fluid isolate. $J$ Clin Microbiol 1995; 33: $1851-1855$.

28. Jensen JS, Hansen HT, Lind K. Isolation of Mycoplasma genitalium from the male urethra. J Clin Microbiol 1996; 34: 286-291.

29. Clyde WA. Mycoplasma species identification based upon growth inhibition by specific antisera. J Immunol 1964; 92: 958-965.

30. Tanner AC, Wu CC. Adaptation of the Sensititre broth microdilution technique to antimicrobial susceptibility testing of Mycoplasma gallisepticum. Avian Dis 1992; 36: 714-717.

31. Hannan PCT, O'Hanlon PJ, Rogers NH. In vitro evaluation of various quinolone antibacterial agents against veterinary mycoplasmas and porcine respiratory bacterial pathogens. Res Vet Sci 1989; 46: 202-211.

32. Schmitt K, Däubener W, Bitter-Suermann D, Hadding U. A safe and efficient method for elimination of cell culture mycoplasmas using ciprofloxacin. J Immunol Methods 1988; 109: $17-25$.

33. Eaton MD, Meicklejohn G, van Herick W. Studies on the etiology of primary atypical pneumonia. A filterable agent transmissable to cotton rats, hamsters, and chick embryos. $J$ Exp Med 1944; 79: 649-668.

34. Eaton MD, Liu C. Studies on sensitivity to streptomycin of the atypical pneumonia agent. J Bacteriol 1957; 74: 784-787.

35. Kubota H. Development of resistance to and dependence on streptomycin in Mycoplasma pneumoniae in vitro. Jpn J Microbiol 1974; 18: $157-163$

36. Niitu Y, Kubota H, Hasegawa S, Komatsu S, Horikawa M, Suetake T. Susceptibility of Mycoplasma pneumoniae to antibiotics in vitro. Jpn J Microbiol 1974; 18: 149-155.

37. Philips I, Shannon K. Aminoglycoside resistance. Br Med Bull 1984; 40: $28-35$.

38. Hannan PCT. Observations on the possible origin of Mycoplasma fermentans incognitus strain based on antibiotic sensitivity tests. J Antimicrob Chemother 1997; 39: 25-30.

39. Hannan PCT, Kearns AM, Sisson PR, Freeman R. Differentiation of strains of $M$. fermentans from various sources by pyrolysis mass spectrometry. $J$ Med Microbiol 1997; 46: $348-353$. 